International Journal of Engineering \& Technology, 7 (4) (2018) $5732-5735$
International Journal of Engineering \& Technology
SPC
Website: www.sciencepubco.com/index.php/IJET
doi: $10.14419 /$ ijet.v7it.14212
Research paper

\title{
Human detection and depth calculation using stereographic vision with pixel disparity and HOG classifier
}

\author{
Vemuri Tejasri ${ }^{1}$ *, D.Ravi Babu ${ }^{2}$, K.N.V. Satyanarayana ${ }^{3}$, D.Srinivas Reddy ${ }^{3}$, R.Shiva Shankar ${ }^{2}$ \\ ${ }^{I}$ M. Tech Scholar, Dept. of CSE, SRKR Engineering College, Chinaamiram, Bhimavaram, A.P., India \\ ${ }^{2}$ Assistant Professor, Dept. of CSE, SRKR Engineering College, Chinaamiram, Bhimavaram, A.P., India \\ ${ }^{3}$ Assistant Professor, Dept. of ECE, SRKR Engineering College, Chinaamiram, Bhimavaram, A.P., India \\ *Corresponding author E-mail: knv.satyan@gmail.com
}

\begin{abstract}
Mainly image processing is used for detection of objects with feasible number of constraints with different detection meth-odologies is defined used by camera-based detection. This method is used to find correspondence with respect to different objects. So that, in this paper, we propose Novel and simple method which is worked based on different region of interests present in video or image. This method mainly worked based on Histogram Oriented Gradients in image processing events. Our method also uses filtering approach with sequential data presentation to access interested data from image or video. Our experimental results mainly show effective visualization results with respect to different selection of regions.
\end{abstract}

Keywords: Microneurosyms; Stereo Vision; Subpixel Disparity; Depth Calculation.

\section{Introduction}

Object detection utilizing picture preparing for the most part incorporates with detection of camera visuals \& identification of different stereo video visuals. This approach uses systems such as protest demonstrate connection [1], segmentation image with different pixels [2], or discovery of particular characteristics such as surface [3] or sequential work representation [4]. Video based vision methodologies have the favorable position of specifically assessing the 3D directions of a picture highlight [5]. This technique distinguishes the frontal region and establishment information and concentrates questions by strategies for PC without human supervision, and moreover a closer recreation to the human acknowledgment. Starting late, stereo vision which can crush various of monocular vision's issues has been thought about. Especially, various stereo calculations which remove 3-dimensional information, particularly the distinction layout, proposed. Hence, various deterrent repression outcomes are in same manner [6]. It merits indicating that all these stereo vision-based inquiry acknowledgment approaches need to find correspondences from the left to the right picture. In this paper, we proposed to find the Region of Interest (ROI) of things like cars, people, bikes utilizing HOG (histogram of Objected Gradients) Descriptor and to recognize corner incorporates into ROI just in the left picture and afterward to make correspondence organizing for these features in the right picture. By this approach, the planning is simply ought to have been enhanced the circumstance outstanding features in the unique district which grows the precision and recuperation the estimation time.

\section{Approach}

The trifling methodologies for human identification and tracking had high computational cost and require particular and costly equipment to work continuously. Different calculations use earlier data about the geometry of the scene, for example, the floor position and the camera alignment to limit the information affiliation and following issues. This approach makes more perplexing the framework establishment and setting, since it is important to figure the camera adjustment and gauge the 3D plane of the floor which thus relies upon the camera area. The greater part of the systems utilized for this issue manage shut world portrayals which depend on a particular learning on the sort of moves making place. This broader case enables us to assess the proposed approach for preparing video streams procured in real world video reconnaissance circumstances. There are different procedures for moving human identification like optical stream, low difference in light, division foundation subtraction, outline distinction and so on. Most accessible methods for distinguishing moving people brought about impediment and were prompting false identifications.

The proposed work of human recognition will be founded on Haar classifiers utilized as calculation. The utilization of Haar classifiers has supported to the update of the framework which is quick and more exact. These techniques permit portioning each picture into an arrangement of locales speaking to the moving people by utilizing a foundation differencing Algorithm.

\section{Related work}

All the more as of late have proposed a neighborhood demonstrating of the background utilizing a blend of K-Gaussian permitting 
for processing video streams with time fluctuating background These techniques give palatable outcomes and can be implemented for continuous preparing without dedicated hardware. In the proposed work the ideas of dynamic template matching and edge differencing have been utilized to implement a strong mechanized human following framework. In this usage a monochrome modern camera has been used to get the video edges and track a human. Utilizing outline differencing on outline by-outline premise a moving human has been distinguished it is followed by employing an effective Template Matching calculation This paper is sorted out as takes after. In area II, the structure of the proposed venture is exhibited where each module must be executed and interfaced In section III, a concise approach with the assistance of flowchart has been demonstrated. In segment IV, comes about are appeared and in the final segment $\mathrm{V}$, finish of the paper is drawn.

\subsection{Exploratory discussion and tools are used}

\subsubsection{Jetson TX1 developer kit}

The NVIDIA Jetson TX1 Developer Kit is a full-highlighted advancement stage for visual figuring. It is perfect for applications requiring high computational execution in a low power envelope. The Jetson TX1Developer Kit is intended to get you up and running rapidly; it comes pre-flashed with a Linux situation, incorporates bolster for some basic APIs, and is upheld by NVIDIA's entire improvement device chain. The board uncovered numerous standard equipment interfaces, empowering a very flexible and extensible stage. For programming refreshes and the engineer SDK supporting your OS picture and host advancement stage, go to http://developer.nvidia.com/inserted figuring The SDK incorporates an OS picture for you to stack onto your gadget, designer apparatuses, supporting documentation, and code tests to enable you to begin. Singular improvement efforts fluctuate and may bring about modifications to the framework configuration. NVIDIA prescribes that you start with the fundamental framework configuration (as transported) to guarantee legitimate framework task before any further advancement.

\subsubsection{Zed camera}

The ZED IS camera that replicates the way human vision works. Using its two EYES and through triangulation, the ZED gives a three-dimensional comprehension of the scene it watches, enabling your application to end up space and movement mindful.

Stereo catch: The ZED is a camera with double lenses.it catch top quality $3 \mathrm{D}$ Video with a wide field of view and out outs two synchronized left and right video streams in next to each other organization on USB 3.0

Profundity perception: Depth observation is the capacity to decide removes amongst items and see the world in three measurements. up to this point, profundity sensors have been constrained to seeing profundity at short range and inside, confining their applications to signal control and body following. Utilizing stereo vision, the ZED is the primary all-inclusive profundity sensors:

- Depth can be caught at longer ranges up to 20M.

- Frame rate of profundity catch can be as high as 100 FPS.

- Field of view is considerably bigger, up to 1000

- The camera works inside and outside, as opposed to dynamic sensors, for example, organized light or time sof flight.

\subsubsection{Positional following}

Utilizing PC vision and stereo SLAM innovation, the ZED additionally comprehends its position and introduction in space, offering full $6 \mathrm{DOF}$ positional following.

In VR/AR, this implies you would now be able to stroll around uninhibitedly and the camera will track the developments anyplace. In case you're into apply autonomy, you would now be able to dependably decide you robots position, introduction, and speed and influence it to explore self-ruling to the directions of decision on a guide. You can get to 6DOF movement following information through the ZED SDK.

\subsubsection{Spatial mapping}

Spatial mapping is the capacity to catch a computerized model of a scene or a protest in the physical world. By consolidating this present reality with the virtual world, it is conceivable to make persuading blended reality experience or robots that understanding their condition.

\subsubsection{Plan procedure}

The plan methods of the proposed channel bank can be described as:

1) For a video chose we recognize the moving individuals by utilizing prepared Haar highlights i.e. the .xml record, which goes about as database for identifying the general population.

2) After the items have been distinguished, following of the identified question is finished utilizing SAD calculation, both location and following must be done all the while.

3) The identified items have been recorded with a specific number so decides the aggregate number of individuals show at a specific purpose of time.

4) Once the quantity of individuals has been evaluated, the distance of individuals from the camera has been resolved in meter as given in condition (4).

\section{Design and Implementation}

\section{1. Histogram of oriented gradient (HOG) technique}

The unique HOG technique presented in 2005 [7], as an element descriptor of picture. The idea of HOG is based on gradient direction; it comprises in determining the histogram of oriented gradient in local community of blob purchased the recognition step.

The criteria actions are provided as follows:

Image pre-processing: first of all, transforming the produced shade blobs into greyish, then using the Gamma modification for normalization, decreasing the lighting impact, and controlling disturbance.

Gradient calculation: determining the gradient of picture in straight and horizontally route of blobs using Sobel advantage owner. The system for the computation is:

$G_{x}(a, b)=H(a+1, b)-H(a-1, b)$

$G_{y}(a, b)=H(a, b+1)-H(a, b-1)$

Where $\mathrm{H}(\mathrm{a}, \mathrm{b})$ is the value of the pixel,

$G_{x}(a, b), G_{y}(a, b)$ are respectively gradients of the straight and horizontally guidelines of pixel $(a, b)$. Therefore, slope scale of the straight and horizontally guidelines a $(a, b)$ of a pixel system are given below

$G(a, b)=\sqrt{G_{X}^{2}(a, b)+G_{Y}^{2}(a, b)}$

$\mathrm{a}(\mathrm{a}, \mathrm{b})=\tan ^{-1}\left(\frac{\mathrm{G}_{\mathrm{y}}(\mathrm{a}, \mathrm{b})}{\mathrm{G}_{\mathrm{y}}(\mathrm{a}, \mathrm{b})}\right)$

Is essential item recognition and detail detecting item is shown in the following flowchart. The flowchart gives an idea about different techniques. 


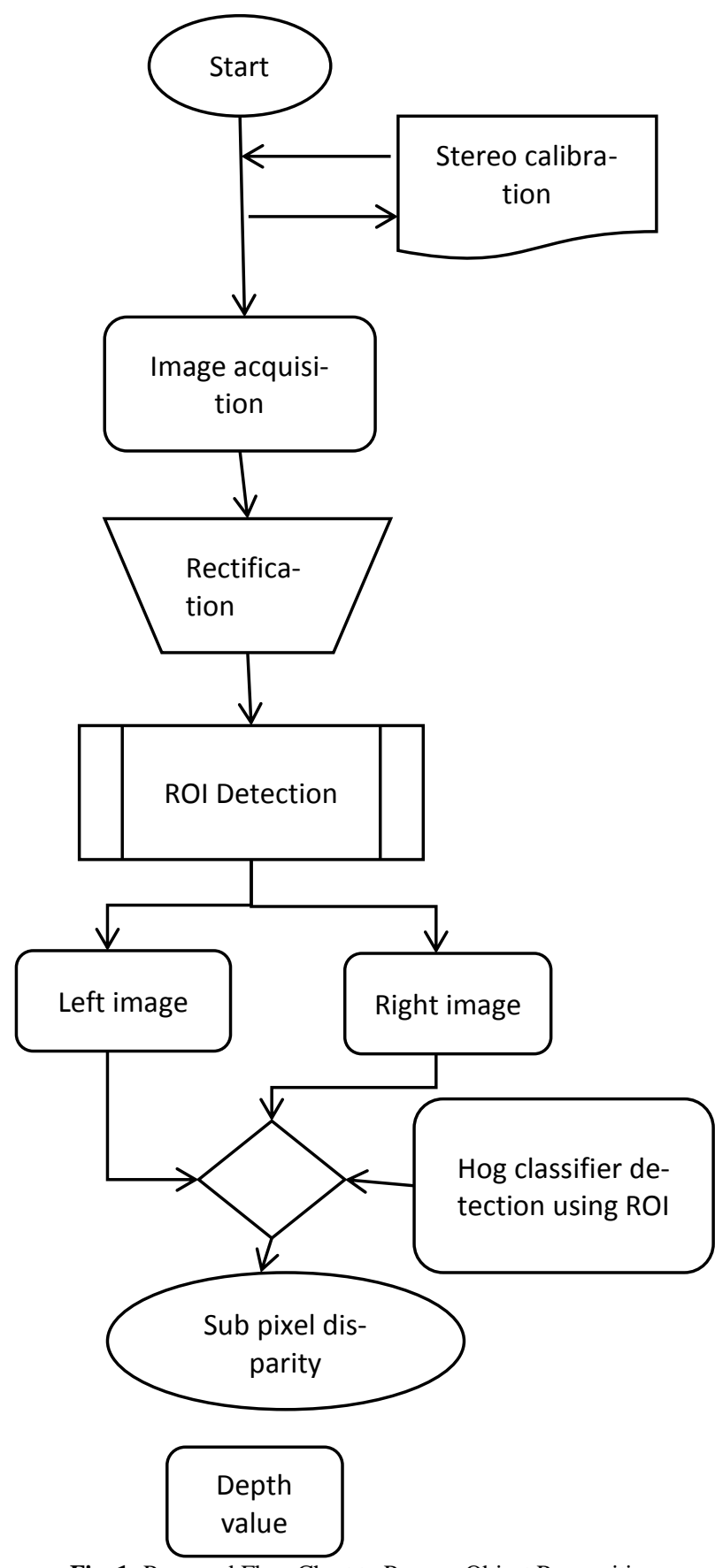

Fig. 1: Proposed Flow Chart to Process Object Recognition.

\section{Results}

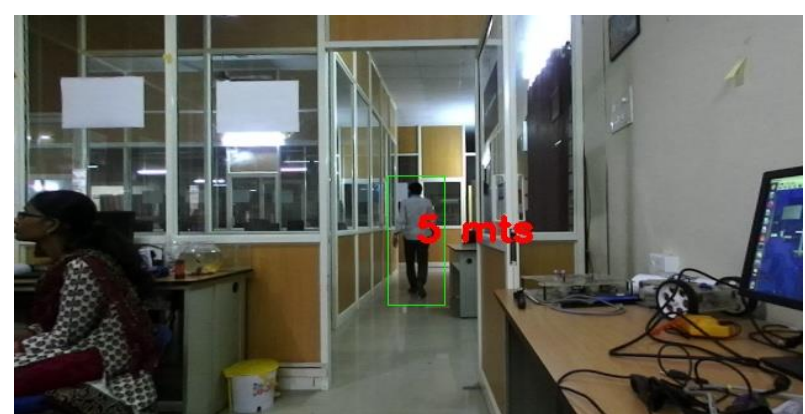

Fig. 2: Object Detection at Distance as $5 \mathrm{mts}$.

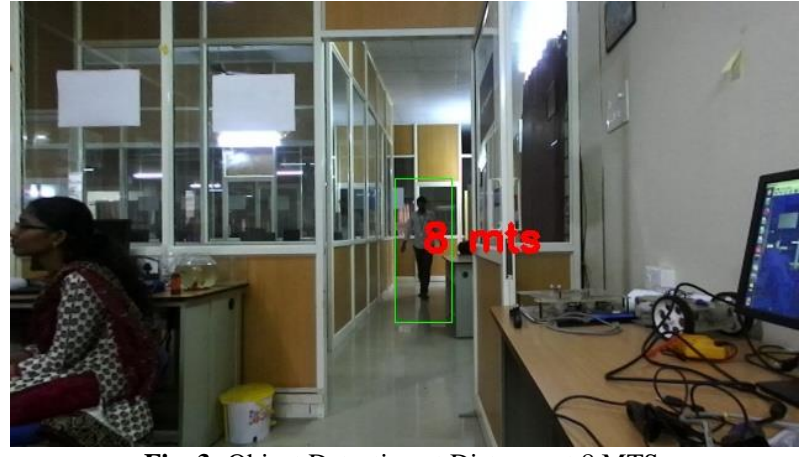

Fig. 3: Object Detection at Distance at 8 MTS

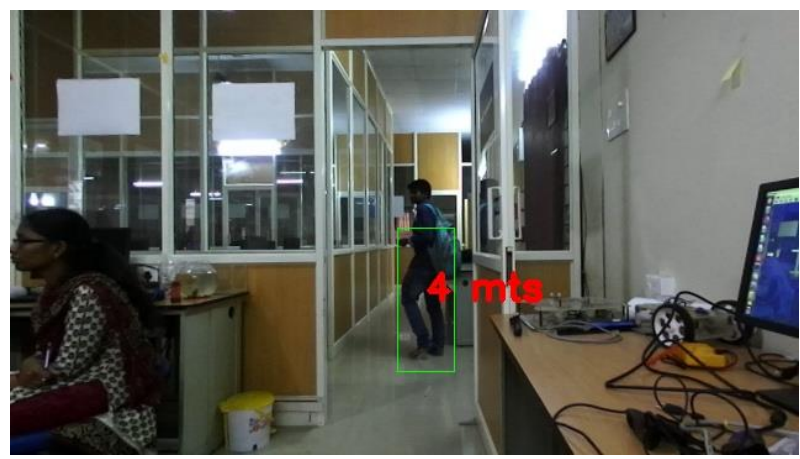

Fig. 4: Objection Tracking at 4 MTS Distance.

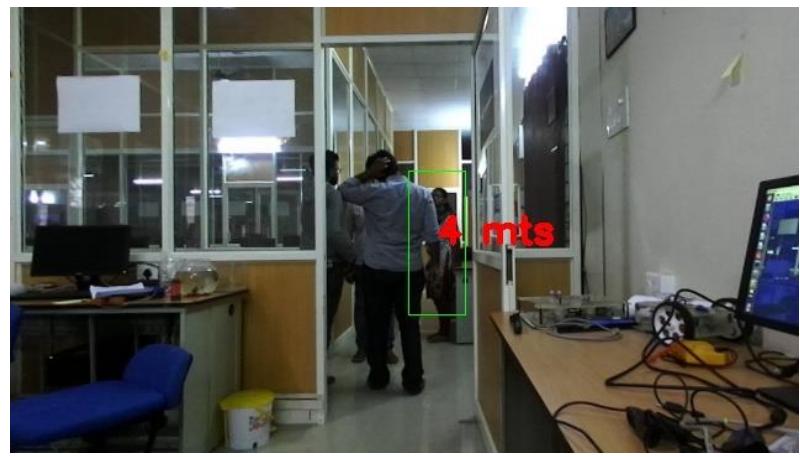

Fig. 5: Multiple Tracking of Objects at Distance of 4 MTS

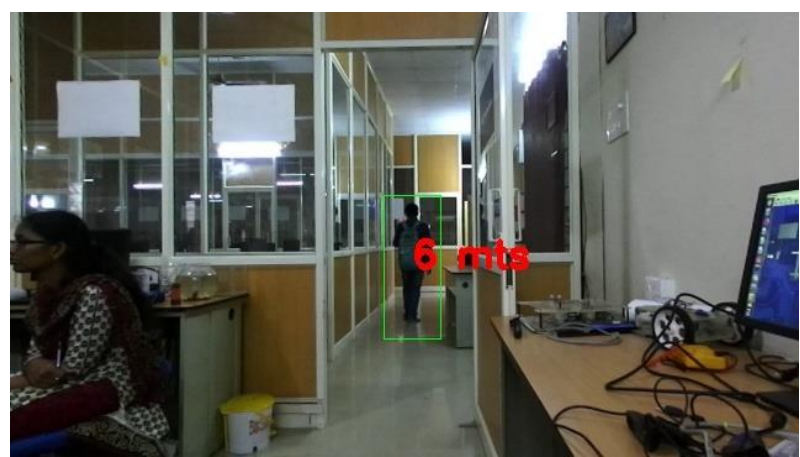

Fig. 6: Moving Object Tracking at Distance of 6 MTS

The object detection and tracking of different lengths are shown from figure 2 to 6 . The figure $2 \& 3$ shows the object detection of

\section{Conclusion}

As per the current issues of customary HOG based order technique a novel two-organize grouping approach is proposed. Two-arrange SVM is chosen to supplant conventional classifier to enhance characterization exactness. At the to begin with phase of grouping, the examples are predominantly isolated into conceivable F\&B human body and not $\mathrm{F} \& \mathrm{~B}$ human body, and after that they were put into the second-arrange characterization among side human 
and non-human to recognize human. At long last the location result is incorporated with the utilization of disjoint sets. The test trial of activity field in mass terminal of Tianjin Port demonstrate that solitary customary SVM calculation has an expansive impediment for F\&B, side and confused stance human body test sets. What's more, it needs flexibility to human stances. The principal organize grouping has a more prominent discovery exactness than the second-organize order, which implies $\mathrm{F} \& \mathrm{~B}$ pose human has more particular HOG highlights than side human. The twoorganize arrangement strategy proposed in this paper has a discovery precision of over $97 \%$, which is higher than utilizing customary SVM. Taking everything into account, the enhanced grouping approach purposed in this paper is more achievable and progressed.

\section{References}

[1] Weilong Song, Guangming Xiong, Linlang Cao, and Yanhua Jiang, "Depth Calculation and Object Detection Using Stereo Vision with Subpixel Disparity and HOG Feature" Intelligent Vehicle Research Center, Beijing Institute of Technology, Beijing, China,100081.

[2] "A Fast HOG Descriptor Using Lookup Table and Integral Image" Chunde Huang \& Jiaxiang Huang, Xiamen University, 422 Siming S Rd, SimingQu, Xiamen, China, 361005xmuhcd@msn.com. \{https://en.wikipedia.org/wiki/Binocular -vision\}.

[3] Gavrila, D.M.: Pedestrian Detection from a Moving Vehicle. In: Vernon, D. (ed.) ECCV 2000. LNCS, vol. 1843, pp. 37-49. Springer, Heidelberg (2000). https://doi.org/10.1007/3-540-45053-X_3.

[4] Ulrich, I., Nourbakhsh, I.: Appearance-Based Obstacle Detection with Monocular Color Vision. In: Proc. of the AAAI National Conference on Artificial Intelligence, Austin, TX (July/August 2000).

[5] Kalinke, T., Tzomakas, C., von Seelen, W.: A Texture based Object Detection and an Adaptive Model-based Classification. In: Procs. IEEE Intelligent Vehicles Symposium 1998, Stuttgart, Germany, pp 341-346 (October 1998).

[6] Kuehnle, A.: Symmetry-based vehicle location for AHS. In: Procs SPIE Transportation Sensors and Controls: Collision Avoidance, Traffic Management, and ITS, Orlando, FL, vol. 2902, pp. 19-27 (November 1998)

[7] Nedevschi, S., Danescu, R., Frentiu, D., Marita, T., Oniga, F., Poco, C.: High Accuracy Stereo Vision System for Far Distance Obstacle Detection. In: IEEE Intelligent Vehicles Symposium, June 14-17. University of Parma, Parma (2004)

[8] Lee, C.-H., Lim, Y.-C., Kwon, S., Lee, J.-H.: Obstacle localization with a binarized v-disparity map using local maximum frequency values in stereo vision. In: International Conference on Signals, Circuits and Systems (2008).

[9] Bradski, G., Kaehler, A.: Learning OpenCV - Computer Vision with the OpenCV Library. O'Reilly Media, Inc., Sebastopol (2008).

[10] Bradski, G., Kaehler, A.: Learning OpenCV - Computer Vision with the Open CV Library. O'Reilly Media, Inc., Sebastopol (2008).

[11] Bouguet, J.-Y.: Visual methods for three-dimensional modeling, PhD Thesis (1999).

[12] Morgan, G.L.K., Liu, J.G., Yan, H.: Precise Subpixel Disparity Measurement from Very Narrow Baseline Stereo. IEEE Transactions on Geoscience and Remote Sensing 48(9), 3424-3433 (2010) https://doi.org/10.1109/TGRS.2010.2046672

[13] Dalal, N.: Finding People in Images and Videos, PhD thesis, France (2006).

[14] Schmid, C., Mohr, R., Bauckhage, C.: Evaluation of interest point detectors. International Journal of Computer Vision 37(2), 151-172 (2000). https://doi.org/10.1023/A:1008199403446. 